\title{
THE SUPREMUM OF CONFORMALLY COVARIANT EIGENVALUES IN A CONFORMAL CLASS
}

\author{
BERND AMMANN, PIERRE JAMMES
}

\begin{abstract}
Let $(M, g)$ be a compact Riemannian manifold of dimension $\geq 3$. We show that there is a metrics $\tilde{g}$ conformal to $g$ and of volume 1 such that the first positive eigenvalue the conformal Laplacian with respect to $\tilde{g}$ is arbitrarily large. A similar statement is proven for the first positive eigenvalue of the Dirac operator on a spin manifold of dimension $\geq 2$.
\end{abstract}

November 11, 2018

\section{CONTENTS}

1. Introduction

2. Preliminaries

3. Asymptotically cylindrical blowups 6

4. Proof of the main theorem 8

References

\section{INTRODUCTION}

The goal of this article is to prove the following theorems.

Theorem 1.1. Let $\left(M, g_{0}, \chi\right)$ be compact Riemannian spin manifold of dimension $n \geq 2$. For any metric $g$ in the conformal class $\left[g_{0}\right]$, we denote the first positive eigenvalue of the Dirac operator on $(M, g, \chi)$ by $\lambda_{1}^{+}\left(D_{g}\right)$. Then

$$
\sup _{g \in\left[g_{0}\right]} \lambda_{1}^{+}\left(D_{g}\right) \operatorname{Vol}(M, g)^{1 / n}=\infty .
$$

Theorem 1.2. Let $\left(M, g_{0}, \chi\right)$ be compact Riemannian manifold of dimension $n \geq 3$. For any metric $g$ in the conformal class $\left[g_{0}\right]$, we denote the first positive eigenvalue of the conformal Laplacian $L_{g}:=$ $\Delta_{g}+\frac{n-2}{4(n-1)} \operatorname{Scal}_{g}$ (also called Yamabe operator) on $(M, g, \chi)$ by $\lambda_{1}^{+}\left(L_{g}\right)$. Then

$$
\sup _{g \in\left[g_{0}\right]} \lambda_{1}^{+}\left(L_{g}\right) \operatorname{Vol}(M, g)^{2 / n}=\infty .
$$

The Dirac operator and the conformal Laplacian belong to a large family of operators, definded in details in subsection 2.3. These operators are called conformally covariant elliptic operators of order $k$ and of bidegree $((n-k) / 2,(n+k) / 2)$, acting on manifolds $(M, g)$ of dimension $n>k$. In particular, our definition includes formal self-adjointness.

The above theorems can be generalized to the following:

\footnotetext{
${ }^{1}$ bernd.ammann at gmx.de, pierre.jammes at univ-avignon.fr
} 
BERND AMMANN, PIERRE JAMMES

Theorem 1.3. Let $P_{g}$ be a conformally covariant elliptic operator of order $k$, of bidegree $((n-k) / 2,(n+$ $k) / 2$ ) acting on manifolds of dimension $n>k$. We also assume that $P_{g}$ is invertible on $\mathbb{S}^{n-1} \times \mathbb{R}($ see Definition 2.4). Let $\left(M, g_{0}\right)$ be compact Riemannian manifold. In the case that $P_{g}$ depends on the spin structure, we assume that $M$ is oriented and is equipped with a spin structure. For any metric $g$ in the conformal class $\left[g_{0}\right]$, we denote the first positive eigenvalue of $P_{g}$ by $\lambda_{1}^{+}\left(P_{g}\right)$. Then

$$
\sup _{g \in\left[g_{0}\right]} \lambda_{1}^{+}\left(P_{g}\right) \operatorname{Vol}(M, g)^{k / n}=\infty .
$$

The interest in this result is motivated by three questions. At first, we found that the infimum

$$
\inf _{g \in\left[g_{0}\right]} \lambda_{1}^{+}\left(D_{g}\right) \operatorname{Vol}(M, g)^{1 / n}
$$

has a rich geometrical structure [4, [2], [7, [6]. In particular it is strictly positive [3] and under some condition preventing the blowup of spheres it is attained [4], [2].

The second motivation comes from comparing this result to results about other differential operators. Let us recall that for the Hodge Laplacian $\Delta_{p}^{g}$ acting on $p$-forms, we have $\sup _{g \in\left[g_{0}\right]} \lambda_{1}\left(\Delta_{p}^{g}\right) \operatorname{Vol}(M, g)^{2 / n}=+\infty$ for $n \geq 4$ and $2 \leq p \leq n-2$ ([17). On the other hand, for the standard Laplacian $\Delta^{g}$ acting on functions, we have $\sup _{g \in\left[g_{0}\right]} \lambda_{k}\left(\Delta^{g}\right) \operatorname{Vol}(M, g)^{2 / n}<+\infty$ (the case $k=1$ is proven in [18] and the general case in [26]). See [24] for a synthetic presentation of this subject.

The essential idea in the proof is to construct metrics with longer and longer cylindrical parts. We will call this an asymptotically cylindrical blowup. Such metrics are also called Pinocchio metrics in [1, 5]. In [1, 5] the behavior of Dirac eigenvalues on such metrics has already been studied partially, but the present article has much stronger results. This provides the third motivation.

Acknowledgements We thank B. Colbois, M. Dahl, E. Humbert and O. Hijazi for many related discussions. We thank R. Gover for some helpful comments on conformally covariant operators, and for several references. The first author wants to thank cordially the Einstein institute at Potsdam-Golm for its hospitality which enabled to write the article.

\section{Preliminaries}

2.1. Notations. In this article $B_{y}(r)$ denotes the ball of radius $r$ around $y, S_{y}(r)=\partial B_{y}(r)$ its boundary. The standard sphere $S_{0}(1) \subset \mathbb{R}^{n}$ in $\mathbb{R}^{n}$ is also denoted by $\mathbb{S}^{n-1}$, its volume is $\omega_{n-1}$. For the volume element of $(M, g)$ we use the notation $d v^{g}$.

For sections $u$ of a vector bundle $V \rightarrow M$ over a Riemannian manifold $(M, g)$ the Sobolev norms $L^{2}$ and $H^{s}, s \in \mathbb{N}$, are defined as

$$
\begin{aligned}
\|u\|_{L^{2}(M, g)}^{2} & :=\int_{M}|u|^{2} d v^{g} \\
\|u\|_{H^{s}(M, g)}^{2} & :=\|u\|_{L^{2}(M, g)}^{2}+\|\nabla u\|_{L^{2}(M, g)}^{2}+\ldots+\left\|\nabla^{s} u\right\|_{L^{2}(M, g)}^{2} .
\end{aligned}
$$

The vector bundle $V$ will be suppressed in the notation. If $M$ and $g$ are clear from the context, we write just $L^{2} H^{s}$. The completions of the compactly supported sections of $V$ with respect to these norms are called $L^{2}(M, g)$ and $H^{s}(M, g)$.

2.2. Removal of singularities. In the proof we will use the following removal of singularity lemma.

Lemma 2.1 (Removal of singularities lemma). Let $\Omega$ be a bounded open subset of $\mathbb{R}^{n}$ containing 0 . Let $P$ be an elliptic differential operator of order $k$ on $\Omega, f \in C^{\infty}(\Omega)$, and let $u \in C^{\infty}(\Omega \backslash\{0\})$ be a solution of

$$
P u=f
$$

on $\Omega \backslash\{0\}$ with

$$
\lim _{\varepsilon \rightarrow 0} \int_{B_{0}(2 \varepsilon)-B_{0}(\varepsilon)}|u| r^{-k}=0 \text { and } \lim _{\varepsilon \rightarrow 0} \int_{B_{0}(\varepsilon)}|u|=0
$$


where $r$ is the distance to 0 . Then $u$ is a (strong) solution of (1) on $\Omega$. The same result holds for sections of vector bundles over relatively compact open subset of Riemannian manifolds.

Proof. We show that $u$ is a weak solution of (11), and then it follows from standard regularity theory, that it is also a strong solution. This means that we have to show that for any given compactly supported smooth test function $\psi: \Omega \rightarrow \mathbb{R}$ we have

$$
\int_{\Omega} u P^{*} \psi=\int_{\Omega} f \psi
$$

Let $\eta: \Omega \rightarrow[0,1]$ be a test function that is identically 1 on $B_{0}(\varepsilon)$, has support in $B_{0}(2 \varepsilon)$, and with $\left|\nabla^{m} \eta\right| \leq C_{m} / \varepsilon^{m}$. It follows that

$$
\sup \left|P^{*}(\eta \psi)\right| \leq C(P, \psi) \varepsilon^{-k}
$$

on $B_{0}(2 \varepsilon) \backslash B_{0}(\varepsilon)$ and $\sup \left|P^{*}(\eta \psi)\right| \leq C(P, \psi)$ on $B_{0}(\varepsilon)$ and hence

$$
\begin{aligned}
\left|\int_{\Omega} u P^{*}(\eta \psi)\right| & \leq C \varepsilon^{-k} \int_{B_{0}(2 \varepsilon) \backslash B_{0}(\varepsilon)}|u|+C \int_{B_{0}(\varepsilon)}|u| \\
& \leq C \int_{B_{0}(2 \varepsilon) \backslash B_{0}(\varepsilon)}|u| r^{-k}+C \int_{B_{0}(\varepsilon)}|u| \rightarrow 0 .
\end{aligned}
$$

We conclude

$$
\begin{aligned}
\int_{\Omega} u P^{*} \psi & =\int_{\Omega} u P^{*}(\eta \psi)+\int_{\Omega} u P^{*}((1-\eta) \psi) \\
& =\underbrace{\int_{\Omega} u P^{*}(\eta \psi)}_{\rightarrow 0}+\underbrace{\int_{\Omega}(P u)(1-\eta) \psi}_{\rightarrow \int_{\Omega} f \psi}
\end{aligned}
$$

for $\varepsilon \rightarrow 0$. Hence the lemma follows.

Condition (2) is obviously satisfied if $\int_{\Omega}|u| r^{-k}<\infty$. It is also satisfied if

$$
\int_{\Omega}|u|^{2} r^{-k}<\infty \text { and } k \leq n
$$

as in this case

$$
\left(\int_{B_{0}(2 \varepsilon) \backslash B_{0}(\varepsilon)}|u| r^{-k}\right)^{2} \leq \int_{\Omega}|u|^{2} r^{-k} \underbrace{\int_{B_{0}(2 \varepsilon) \backslash B_{0}(\varepsilon)} r^{-k}}_{\leq C} .
$$

2.3. Conformally covariant elliptic operators. In this subsection we present a class of certain conformally covariant elliptic operators. Many important geometric operators are in this class, in particular the conformal Laplacian, the Paneitz operator, the Dirac operator, see also [19, 16, 20, for more examples.

Such an operator is not just one single differential operator, but a procedure how to associate to an $n$-dimensional Riemannian manifold $(M, g)$ (potentially with some additional structure) a differential operator $P_{g}$ of order $k$ acting on a vector bundle. The important fact is that if $g_{2}=f^{2} g_{1}$, then one claims

$$
P_{g_{2}}=f^{-\frac{n+k}{2}} P_{g_{1}} f^{\frac{n-k}{2}} .
$$

One also expresses this by saying that $P$ has bidegree $((n-k) / 2,(n+k) / 2)$.

The sense of this equation is apparent if $P_{g}$ is an operator from $C^{\infty}(M)$ to $C^{\infty}(M)$. If $P_{g}$ acts on a vector bundle or if some additional structure (as e.g. spin structure) is used for defining it, then a rigorous and careful definition needs more attention. The language of categories provides a good formal framework 27. The concept of conformally covariant elliptic operators is already used by many authors, but we do not know of a reference where a formal definition is carried out that fits to our context. (See 25] for a similar categorial approach that includes some of the operators presented here.) Often an intuitive definition is 
used. The intuitive definition is obviously sufficient if one deals with operators acting on functions, such as the conformal Laplacian or the Paneitz operator. However to properly state Theorem 1.3 we need the following definition.

Let Riem $^{n}$ (resp. Riemspin ${ }^{n}$ ) be the category $n$-dimensional Riemannian manifolds (resp. $n$-dimensional Riemannian manifolds with orientation and spin structure). Morphisms from $\left(M_{1}, g_{1}\right)$ to $\left(M_{2}, g_{2}\right)$ are conformal embeddings $\left(M_{1}, g_{1}\right) \hookrightarrow\left(M_{2}, g_{2}\right)$ (resp. conformal embeddings preserving orientation and spin structure).

Let Laplace $_{k}^{n}$ (resp. Dirac $\left.{ }_{k}^{n}\right)$ be the category whose objects are $\left\{(M, g), V_{g}, P_{g}\right\}$ where $(M, g)$ in an object of Riem $^{n}$ (resp. Riemspin ${ }^{n}$ ), where $V_{g}$ is a vector bundle with a scalar product on the fibers, where $P_{g}: \Gamma\left(V_{g}\right) \rightarrow \Gamma\left(V_{g}\right)$ is an elliptic formally selfadjoint differential operator of order $k$.

A morphism $(\iota, \kappa)$ from $\left\{\left(M_{1}, g_{1}\right), V_{g_{1}}, P_{g_{1}}\right\}$ to $\left\{\left(M_{2}, g_{2}\right), V_{g_{2}}, P_{g_{2}}\right\}$ consists of a conformal embedding $\iota:\left(M_{1}, g_{1}\right) \hookrightarrow\left(M_{2}, g_{2}\right)$ (preserving orientation and spin structure in the case of Dirac $c_{k}^{n}$ ) together with a fiber isomorphism $\kappa: \iota^{*} V_{g_{2}} \rightarrow V_{g_{1}}$ perserving fiberwise length, such that $P_{g_{1}}$ and $P_{g_{2}}$ satisfy the conformal covariance property (6). For stating this property precisely, let $f>0$ be defined by $\iota^{*} g_{2}=f^{2} g_{1}$, and let $\kappa_{*}: \Gamma\left(V_{g_{2}}\right) \rightarrow \Gamma\left(V_{g_{1}}\right), \kappa_{*}(\varphi)=\kappa \circ \varphi \circ \iota$. Then the conformal covariance property is

$$
\kappa_{*} P_{g_{2}}=f^{-\frac{n+k}{2}} P_{g_{1}} f^{\frac{n-k}{2}} \kappa_{*} .
$$

In the following the maps $\kappa$ and $\iota$ will often be evident from the context and then will be omitted. The transformation formula (7) then simplifies to (6).

Definition 2.2. A conformally covariant elliptic operator of order $k$ and of bidegree $((n-k) / 2,(n+k) / 2)$ is a contravariant functor from Riem $^{n}$ (resp. Riemspin ${ }^{n}$ ) to Laplace $_{k}^{n}$ (resp. Dirac ${ }_{k}^{n}$ ), mapping $(M, g$ ) to $\left(M, g, V_{g}, P_{g}\right)$ in such a way that the coefficients are continuous in the $C^{k}$-topology of metrics (see below). To shorten notation, we just write $P_{g}$ or $P$ for this functor.

It remains to explain the $C^{k}$-continuity of the coefficients.

For Riemannian metrics $g, g_{1}, g_{2}$ defined on a compact set $K \subset M$ we set

$$
d_{C^{k}(K)}^{g}\left(g_{1}, g_{2}\right):=\max _{t=0, \ldots, k}\left\|\left(\nabla_{g}\right)^{t}\left(g_{1}-g_{2}\right)\right\|_{C^{0}(K)} .
$$

For a fixed background metric $g$, the relation $d_{C^{k}(K)}^{g}(\cdot, \cdot)$ defines a distance function on the space of metrics on $K$. The topology induced by $d^{g}$ is independent of this background metric and it is called the $C^{k}$-topology of metrics on $K$.

Definition 2.3. We say that the coefficients of $P$ are continuous in the $C^{k}$-topology of metrics if for any metric $g$ on a manifold $M$, and for any compact subset $K \subset M$ there is a neighborhood $\mathcal{U}$ of $\left.g\right|_{K}$ in the $C^{k}$-topology of metrics on $K$, such that for all metrics $\tilde{g},\left.\tilde{g}\right|_{K} \in \mathcal{U}$, there is an isomorphism of vector bundles $\hat{\kappa}:\left.\left.V_{g}\right|_{K} \rightarrow V_{\tilde{g}}\right|_{K}$ over the identity of $K$ with induced map $\hat{\kappa}_{*}: \Gamma\left(\left.V_{g}\right|_{K}\right) \rightarrow \Gamma\left(\left.V_{\tilde{g}}\right|_{K}\right)$ with the property that the coefficients of the differential operator

$$
P_{g}-\left(\hat{\kappa}_{*}\right)^{-1} P_{\tilde{g}} \hat{\kappa}_{*}
$$

depend continuously on $\tilde{g}$ (with repsect to the $C^{k}$-topology of metrics).

2.4. Invertibility on $\mathbb{S}^{n-1} \times \mathbb{R}$. Let $P$ be a conformally covariant elliptic operator of order $k$ and of bidegree $((n-k) / 2,(n+k) / 2)$. For $(M, g)=\mathbb{S}^{n-1} \times \mathbb{R}$, the operator $P_{g}$ is a self-adjoint operator $H^{k} \subset L^{2} \rightarrow L^{2}$ (see Lemma 3.1 and the comments thereafter).

Definition 2.4. We say that $P$ is invertible on $\mathbb{S}^{n-1} \times \mathbb{R}$ if $P_{g}$ is an invertible operator $H^{k} \rightarrow L^{2}$ where $g$ is the standard product metric on $\mathbb{S}^{n-1} \times \mathbb{R}$. In order words there is a constant $\sigma>0$ such that the spectrum of $P_{g}: \Gamma_{H^{k}}\left(V_{g}\right) \rightarrow \Gamma_{L^{2}}\left(V_{g}\right)$ is contained in $(-\infty,-\sigma] \cup[\sigma, \infty)$ for any $g \in U$. In the following, the largest such $\sigma$ will be called $\sigma_{P}$.

We conjecture that any conformally covariant elliptic operator of order $k$ and of bidegree $((n-k) / 2,(n+$ $k) / 2$ ) with $k<n$ is invertible on $\mathbb{S}^{n-1} \times \mathbb{R}$. 


\subsection{Examples.}

Example 1: The Conformal Laplacian

Let

$$
L_{g}:=\Delta_{g}+\frac{n-2}{4(n-1)} \operatorname{Scal}_{g}
$$

be the conformal Laplacian. It acts on functions on a Riemannian manifold $(M, g)$, i.e. $V_{g}$ is the trivial real line bundle $\underline{\mathbb{R}}$. Let $\iota:\left(M_{1}, g_{1}\right) \hookrightarrow\left(M_{2}, g_{2}\right)$ be a conformal embedding. Then we can choose $\kappa:=\mathrm{Id}: \iota^{*} V_{g_{2}} \rightarrow V_{g_{1}}$ and formula (7) holds for $k=2$ (see e.g. 13, Section 1.J]). All coefficients of $L_{g}$ depend continuously on $g$ in the $C^{2}$-topology. Hence $L$ is a conformally covariant elliptic operator of order 2 and of bidegree $((n-2) / 2,(n+2) / 2)$.

The scalar curvature of $\mathbb{S}^{n-1} \times \mathbb{R}$ is $(n-1)(n-2)$. Hence the spectrum of $L_{g}$ on $\mathbb{S}^{n-1} \times \mathbb{R}$ of $L_{g}$ coincides with the essential spectrum of $L_{g}$ and is $\left[\sigma_{L}, \infty\right)$ with $\sigma_{L}:=(n-2)^{2} / 4$. Hence $L$ is invertible on $S^{n-1} \times \mathbb{R}$ if (and only if) $n>2$.

Example 2: The Paneitz operator

Let $(M, g)$ be a smooth, compact Riemannian manifold of dimension $n \geq 5$. The Paneitz operator $P_{g}$ is given by

$$
P_{g} u=\left(\Delta_{g}\right)^{2} u-\operatorname{div}_{g}\left(A_{g} d u\right)+\frac{n-4}{2} Q_{g} u
$$

where

$$
\begin{gathered}
A_{g}:=\frac{(n-2)^{2}+4}{2(n-1)(n-2)} \operatorname{Scal}_{g} g-\frac{4}{n-2} \operatorname{Ric}_{g}, \\
Q_{g}=\frac{1}{2(n-1)} \Delta_{g} \operatorname{Scal}_{g}+\frac{n^{3}-4 n^{2}+16 n-16}{8(n-1)^{2}(n-2)^{2}} \operatorname{Scal}_{g}^{2}-\frac{2}{(n-2)^{2}}\left|\mathrm{Ric}_{g}\right|^{2} .
\end{gathered}
$$

This operator was defined by Paneitz in the case $n=4$, and it was generalized by Branson in 15 . to arbitrary dimensions $\geq 4$. We also refer to Theorem 1.21 of the overview article [14]. The explicit formula presented above can be found e.g. in [21. The coefficients of $P_{g}$ depend continuous on $g$ in the $C^{4}$-topology

As in the previous example we can choose for $\kappa$ the identity, and then the Paneitz operator $P_{g}$ is a conformally covariant elliptic operator of order 4 and of bidegree $((n-4) / 2,(n+4) / 2)$.

On $\mathbb{S}^{n-1} \times \mathbb{R}$ one calculates

$$
A_{g}:=\frac{(n-4) n}{2} \mathrm{Id}+4 \pi_{\mathbb{R}}>0
$$

where $\pi_{\mathbb{R}}$ is projection to vectors parallel to $\mathbb{R}$.

$$
Q_{g}:=\frac{(n-4) n^{2}}{8} .
$$

We conclude

$$
\sigma_{P}=Q=\frac{(n-4) n^{2}}{8}
$$

and $P$ is invertible on $\mathbb{S}^{n-1} \times \mathbb{R}$ if (and only if) $n>4$.

Examples 3: The Dirac operator.

Let $\tilde{g}=f^{2} g$. Let $\Sigma^{g} M$ resp. $\Sigma^{\tilde{g}} M$ be the spinor bundle of $(M, g)$ resp. $(M, \tilde{g})$. Then there is a fiberwise isomorphism $\beta_{\tilde{g}}^{g}: \Sigma^{g} M \rightarrow \Sigma^{\tilde{g}} M$, preserving the norm such that

$$
D_{\tilde{g}} \circ \beta_{\tilde{g}}^{g}(\varphi)=f^{-\frac{n+1}{2}} \beta_{\tilde{g}}^{g} \circ D_{g}\left(f^{\frac{n-1}{2}} \varphi\right),
$$

see [23, 12, 22] for details. Furthermore, the cocycle conditions

$$
\beta_{\tilde{g}}^{g} \circ \beta_{g}^{\tilde{g}}=\mathrm{Id} \quad \text { and } \quad \beta_{g}^{\hat{g}} \circ \beta_{\hat{g}}^{\tilde{g}} \circ \beta_{\tilde{g}}^{g}=\mathrm{Id}
$$


hold for conformal metrics $g, \tilde{g}$ and $\hat{g}$. We will hence use the map $\beta_{\tilde{g}}^{g}$ to identify $\Sigma^{g} M$ with $\Sigma^{\tilde{g}} M$. Hence we simply get

$$
D_{\tilde{g}} \varphi=f^{-\frac{n+1}{2}} \circ D_{g}\left(f^{\frac{n-1}{2}} \varphi\right) .
$$

All coefficients of $D_{g}$ depend continuously on $g$ in the $C^{1}$-topology. Hence $D$ is a conformally covariant elliptic operator of order 1 and of bidegree $((n-1) / 2,(n+1) / 2)$.

The Dirac operator on $\mathbb{S}^{n-1} \times \mathbb{R}$ can be decomposed as $D_{\text {vert }}+D_{\text {hor }}$, where the first part is the sum over the derivations (and Clifford multiplication) along $\mathbb{S}^{n-1}$ and where $D_{\text {hor }}=\partial_{t} \cdot \nabla_{\partial_{t}}$, where $\partial_{t} \cdot$ is Clifford multiplication with $\partial_{t}, t \in \mathbb{R}$. $D_{\text {vert }}$ and $D_{\text {hor }}$ anticommute. The spectrum of $D_{\text {vert }}$ is just the spectrum of the Dirac operator on $\mathbb{S}^{n-1}$, and hence we see with [1]

$$
\operatorname{spec} D_{\text {vert }}=\left\{ \pm\left(\frac{n-1}{2}+k\right) \mid k \in \mathbb{N}_{0}\right\} .
$$

The operator $\left(D_{\text {hor }}\right)^{2}$ is the ordinary Laplacian on $\mathbb{R}$ and hence has spectrum $[0, \infty)$. Together this implies that the spectrum of the Dirac operator on $\mathbb{S}^{n-1} \times \mathbb{R}$ is $\left(-\infty,-\sigma_{D}\right] \cup\left[\sigma_{D}, \infty\right)$ with $\sigma_{D}=\frac{n-1}{2}$.

Hence $D$ is invertible on $S^{n-1} \times \mathbb{R}$ if (and only if) $n>1$.

In the case $n=2$ these statements are only correct if the circle $\mathbb{S}^{n-1}=\mathbb{S}^{1}$ carries the spin structure induced from the ball. In our article all circles $\mathbb{S}^{1}$ carry this bounding spin structure due to the geometry of the asymptotically cylindrical blowups.

Example 4: The Rarita-Schwinger operator and many other Fegan type operators are conformally covariant elliptic operators of order 1 and of bidegree $((n-1) / 2,(n+1) / 2)$. See [19] and in the work of T. Branson for more information.

Example 5: Assume that $(M, g)$ is a Riemannian spin manifold that carries a vector bundle $W \rightarrow M$ with metric and metric connection. Then there is a natural first order operator $\Gamma(\Sigma M \otimes W) \rightarrow \Gamma(\Sigma M \otimes W)$, the Dirac operator twisted by $W$. This operator has similar properties as conformally covariant elliptic operators of order 1 and of bidegree $((n-1) / 2,(n+1) / 2)$. The methods of our article can be easily adapted in order to show that Theorem 1.3 is also true for this twisted Dirac operator. However, twisted Dirac operators are not "conformally covariant elliptic operators" in the above sense. They could have been included in this class by replacing the category Riemspin ${ }^{n}$ by a category of Riemannian spin manifolds with twisting bundles. In order not to overload the formalism we chose not to present these larger categories.

The same discussion applies to the $\operatorname{spin}^{\mathrm{c}}$-Dirac operator of a spin ${ }^{\mathrm{c}}$-manifold.

\section{Asymptotically CYLINDRICAL BLOWUPS}

3.1. Convention. From now on we suppose that $P_{g}$ is a conformally covariant elliptic operator of order $k$, of bidegree $((n-k) / 2,(n+k) / 2)$, acting on manifolds of dimension $n$ and invertible on $\mathbb{S}^{n-1} \times \mathbb{R}$.

3.2. Definition of the metrics. Let $g_{0}$ be a Riemannian metric on a compact manifold $M$. We can suppose that the injectivity radius in a fixed point $y \in M$ is larger than 1 . The geodesic distance from $y$ to $x$ is denoted by $d(x, y)$.

We choose a smooth function $F_{\infty}: M \backslash\{y\} \rightarrow[1, \infty)$ such such that $F_{\infty}(x)=1$ if $d(x, y) \geq 1, F_{\infty}(x) \leq 2$ if $d(x, y) \geq 1 / 2$ and such that $F_{\infty}(x)=d(x, y)^{-1}$ if $d(x, y) \in(0,1 / 2]$. Then for $L \geq 1$ we define $F_{L}$ to be a smooth positive function on $M$, depending only on $d(x, y)$, such that $F_{L}(x)=F_{\infty}(x)$ if $d(x, y) \geq e^{-L}$ and $F_{L}(x) \leq d(x, y)^{-1}=F_{\infty}(x)$ if $d(x, y) \leq e^{-L}$.

For any $L \geq 1$ or $L=\infty$ set $g_{L}:=F_{L}^{2} g_{0}$. The metric $g_{\infty}$ is a complete metric on $M_{\infty}$.

The family of metrics $\left(g_{L}\right)$ is called an asymptotically cylindrical blowup, in the literature it is denoted as a family of Pinocchio metrics [5], see also Figure 1. 

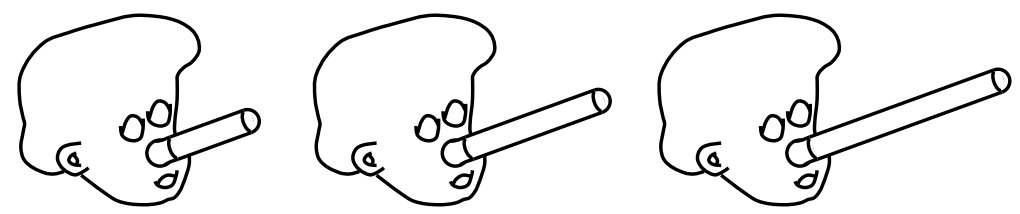

Figure 1. Asymptotically cylindrical metrics $g_{L}$ (alias Pinocchio metrics) with growing nose length $L$.

3.3. Eigenvalues and basic properties on $\left(M, g_{L}\right)$. For the $P$-operator associated to $\left(M, g_{L}\right), L \in$ $\{0\} \cup[1, \infty)$ (or more exactly its selfadjoint extension) we simply write $P_{L}$ instead of $P_{g_{L}}$. As $M$ is compact the spectrum of $P_{L}$ is discrete.

We will denote the spectrum of $P_{L}$ in the following way

$$
\ldots \leq \lambda_{1}^{-}\left(P_{g_{L}}\right)<0=0 \ldots=0<\lambda_{1}^{+}\left(P_{g_{L}}\right) \leq \lambda_{2}^{+}\left(P_{g_{L}}\right) \leq \ldots,
$$

where each eigenvalue appears with the multiplicity of the multiplicity of the eigenspace. The zeros might appear on this list or not, depending on whether $P_{g_{L}}$ is invertible or not. The spectrum might be entirely positive (for example the conformal Laplacian $Y_{g}$ on the sphere) in which case $\lambda_{1}^{-}\left(P_{g_{L}}\right)$ is not defined. Similarly, $\lambda_{1}^{+}\left(P_{g_{L}}\right)$ is not defined if the spectrum of $\left(P_{g_{L}}\right)$ is negative.

3.4. The asymptotic analysis of $\left(M_{\infty}, g_{\infty}\right)$. The asymptotic analysis of non-compact manifolds as $\left(M_{\infty}, g_{\infty}\right)$ is more complicated than in the compact case. Nevertheless $\left(M_{\infty}, g_{\infty}\right)$ is an asymptotically cylindrical manifolf for there exists nowadays an extensive literature. We will need only very few of these properties that will be summarized in this subsection. Proofs will only be sketched.

Different approaches can be used for the proof. The following lemma shows that $\left(M_{\infty}, g_{\infty}\right)$ carries a $b$-structure in the sense of Melrose. "Manifolds with $b$-structures" form a subclass of "Manifolds with a Lie structure at infinity", also called "Lie manifolds" [9, [10], [8]. We choose to use Melrose's b-calculus 28 in this article as this calculus is more widely known, but similar statements hold in the larger category of "Manifolds with a Lie structure at infinity".

Lemma 3.1. The manifold $\left(M_{\infty}, g_{\infty}\right)$ is an exact b-metric in the sense of [28, Def. 2.8].

Proof. Let $S_{y} M$ be the unit tangent bundle at $y$. For any $X \in S_{y} M$ let $\gamma_{X}$ be the geodesic with $\dot{\gamma}(0)=X$. Then for small $\varepsilon>0$ the map $\Phi: S_{y} M \times[0, \varepsilon) \rightarrow M, \quad(X, t) \mapsto \gamma_{X}(t)$ is smooth and a diffeomorphism from $S_{y} M \times(0, \varepsilon)$ to $B_{y}(\varepsilon) \backslash\{0\}$. We define $\bar{M}:=M_{\infty} \cup: S_{y} M \times[0, \varepsilon) / \sim$ where $\sim$ indicates that $(X, t) \in S_{y} M \times(0, \varepsilon)$ is glued together with $\Phi(X, t) . \bar{M}$ is a manifold with boundary $S_{y} M$ and interior $M_{\infty}$. Expressing the metric in normal coordinates, one sees that $d(x, y)^{2} g$ extends to an exact $b$-metric on $\bar{M}$.

From this observation may properties already follow with standard arguments similarly as in the compact case. The operator $P_{g_{\infty}}$ has a self-adjoint extension, denoted by $P_{\infty}$. The essential spectrum of $P_{\infty}$ coincides with the essential spectrum of the $P$-operator on the standard cylinder $\mathbb{S}^{n-1} \times \mathbb{R}$ which is contained in $\left(-\infty,-\sigma_{P}\right] \cup\left[\sigma_{P}, \infty\right)$. Hence the spectrum of $P_{\infty}$ in the interval $\left(-\sigma_{P}, \sigma_{P}\right)$ is discrete as well. Eigenvalues of $P_{\infty}$ in this interval will be called small eigenvalues of $P_{\infty}$. Similarly we use the notation $\lambda_{j}^{ \pm}\left(P_{\infty}\right)$ for the small eigenvalues of $P_{\infty}$.

Proposition 3.2. Let $P$ be a conformally covariant elliptic operator. Then on $\left(M_{\infty}, g_{\infty}\right)$ we have

$$
\left\|\left(\nabla^{\infty}\right)^{s} u\right\|_{L^{2}\left(g_{\infty}\right)} \leq C\left(\|u\|_{L^{2}\left(g_{\infty}\right)}+\left\|P_{\infty} u\right\|_{L^{2}\left(g_{\infty}\right)}\right)
$$


for all $s \in\{0,1, \ldots, k\}$.

Proof. Choose a $\lambda \in \mathbb{R}$ that is not in the spectrum of $P$. The continuity of the coefficients of $P$ together with the fact that $P_{g_{0}}$ extends to $M$ implies that $P_{g_{\infty}}$ is an operator compatible with the $b$-structure. Hence we we can apply [28, Proposition] for $Q:=P-\lambda$. We see that $P-\lambda$ is an isomorphism from $H^{k}$ to $L^{2}$. Hence a constant $C>0$ exists with

$$
C\|(P-\lambda) u\|_{L^{2}} \geq\|u\|_{H^{k}} .
$$

using the triangle inequality we get

$$
\|u\|_{H^{k}} \leq C \lambda\|u\|_{L^{2}\left(g_{\infty}\right)}+\left\|P_{\infty} u\right\|_{L^{2}\left(g_{\infty}\right)}
$$

which is equivalent to the statement.

3.5. The kernel. Having recalled these previously known facts we will now study the kernel of the conformally covariant operators.

If $g$ and $\tilde{g}=f^{2}$ are conformal metrics on a compact manifold $M$, then

$$
\varphi \mapsto f-\frac{n-k}{2} \varphi
$$

obviously defines an isomorphism from $\operatorname{ker} P_{g}$ to $\operatorname{ker} P_{\tilde{g}}$. It is less obvious that a similar statement holds if we compare $g_{0}$ and $g_{\infty}$ defined before:

Proposition 3.3. The map

$$
\begin{aligned}
\operatorname{ker} P_{0} & \rightarrow \operatorname{ker} P_{\infty} \\
\varphi_{0} & \mapsto \varphi_{\infty}=F_{\infty}^{-\frac{n-k}{2}} \varphi_{0}
\end{aligned}
$$

is an isomorphism of vector spaces.

Proof. Suppose $\varphi_{0} \in \operatorname{ker} P_{0}$. Using standard regularity results it is clear that $\sup \left|\varphi_{0}\right|<\infty$. Then

$$
\begin{aligned}
\int_{M_{\infty}}\left|\varphi_{\infty}\right|^{2} d v^{g_{\infty}} & \leq \int_{M \backslash B_{y}(1 / 2)}\left|\varphi_{\infty}\right|^{2} d v^{g_{\infty}}+\sup \left|\varphi_{0}\right|^{2} \int_{B_{y}(1 / 2)} F_{\infty}^{-(n-k)} d v^{g_{\infty}} \\
& \leq 2^{k} \int_{M \backslash B_{y}(1 / 2)}\left|\varphi_{0}\right|^{2} d v^{g_{0}}+\sup \left|\varphi_{0}\right|^{2} \omega_{n-1} \int_{0}^{1 / 2} \frac{r^{n-1}}{r^{k}} d r<\infty .
\end{aligned}
$$

Furthermore, formula (6) implies $P_{\infty} \varphi_{\infty}=0$. Hence the map is well-defined. In order to show that it is an isomorphism we show that the obvious inverse $\varphi_{\infty} \mapsto \varphi_{0}:=F_{\infty}^{\frac{n-k}{2}} \varphi_{\infty}$ is well defined. To see this we start with an $L^{2}$-section in the kernel of $P_{\infty}$.

We calculate

$$
\int_{M} F_{\infty}^{k}\left|\varphi_{0}\right|^{2} d v^{g_{0}}=\int_{M_{\infty}}\left|\varphi_{\infty}\right|^{2} d v^{g_{\infty}}
$$

Using again (6) we see that this section satisfies $P_{0} \varphi_{0}$ on $M \backslash\{y\}$. Hence condition (5) is satisfied, and together with the removal of singularity lemma (Lemma 2.1) one obtains that the inverse map is well-defined. The proposition follows.

\section{Proof of the Main theorem}

4.1. Stronger version of the main theorem. We will now show the following theorem.

Theorem 4.1. Let $P$ be a conformally covariant elliptic operator of order $k$, of bidegree $((n-k) / 2,(n+$ $k) / 2$ ), on manifolds of dimension $n>k$. We assume that $P$ is invertible on $\mathbb{S}^{n-1} \times \mathbb{R}$.

If $\liminf \operatorname{in}_{L \rightarrow \infty}\left|\lambda_{j}^{ \pm}\left(P_{L}\right)\right|<\sigma_{P}$, then

$$
\lambda_{j}^{ \pm}\left(P_{L}\right) \rightarrow \lambda_{j}^{ \pm}\left(P_{\infty}\right) \in\left(-\sigma_{P}, \sigma_{P}\right) \quad \text { for } L \rightarrow \infty .
$$


In the case $\operatorname{Spec}\left(P_{g_{0}}\right) \subset(0, \infty)$ the theorem only makes a statement about $\lambda_{j}^{+}$, and conversely in the case that $\operatorname{Spec}\left(P_{g_{0}}\right) \subset(-\infty, 0)$ it only makes a statement about $\lambda_{j}^{-}$.

Obviously this theorem implies Theorem 1.3 .

4.2. The supremum part of the proof of Theorem 4.1, At first we prove that

$$
\limsup _{L \rightarrow \infty}\left(\lambda_{j}^{+}\left(P_{L}\right)\right) \leq \lambda_{j}^{+}\left(P_{\infty}\right) .
$$

Let $\varphi_{1}, \ldots, \varphi_{j}$ be sequence of $L^{2}$-orthonormal eigenvectors of $P_{\infty}$ to eigenvalues $\lambda_{1}^{+}\left(P_{\infty}\right), \ldots, \lambda_{j}^{+}\left(P_{\infty}\right) \in$ $[-\bar{\lambda}, \bar{\lambda}], \bar{\lambda}<\sigma_{P}$. We choose a cut-off function $\chi: M \rightarrow[0,1]$ with $\chi(x)=1$ for $-\log (d(x, y)) \leq T$, $\chi(y)=0$ for $-\log (d(x, y)) \geq 2 T$, and $\left|\left(\nabla^{\infty}\right)^{s} \chi\right|_{g_{\infty}} \leq C_{s} / T^{s}$ for all $s \in\{0, \ldots, k\}$.

Let $\varphi$ be a linear combination of the eigenvectors $\varphi_{1}, \ldots, \varphi_{j}$. From Proposition 3.2 we see that

$$
\left\|\left(\nabla^{\infty}\right)^{s} \varphi\right\|_{L^{2}\left(M_{\infty}, g_{\infty}\right)} \leq C\|\varphi\|_{L^{2}\left(M_{\infty}, g_{\infty}\right)}
$$

where $C$ only depends on $\left(M_{\infty}, g_{\infty}\right)$. Hence for sufficiently large $T$

$$
\left\|P_{\infty}(\chi \varphi)-\chi P_{\infty} \varphi\right\|_{L^{2}\left(M_{\infty}, g_{\infty}\right)} \leq k C / T\|\varphi\|_{L^{2}\left(M_{\infty}, g_{\infty}\right)} \leq 2 k C / T\|\chi \varphi\|_{L^{2}\left(M_{\infty}, g_{\infty}\right)}
$$

for sufficiently large $T$ as $\|\chi \varphi\|_{L^{2}\left(M_{\infty}, g_{\infty}\right)} \rightarrow\|\varphi\|_{L^{2}\left(M_{\infty}, g_{\infty}\right)}$ for $T \rightarrow \infty$. The section $\chi \varphi$ can be interpreted as a section on $\left(M, g_{L}\right)$ if $L>2 T$, and on the support of $\chi \varphi$ we have $g_{L}=g_{\infty}$ and $P_{\infty}(\chi \varphi)=P_{L}(\chi \varphi)$. Hence standard Rayleigh quotient arguments imply that if $P_{\infty}$ has $m$ eigenvalues (counted with mulitplicity) in the intervall $[a, b]$ then $P_{L}$ has $m$ eigenvalues in the intervall $[a-2 k C / T, b+2 k C / T]$. Taking the limit $T \rightarrow \infty$ we obtain (10).

By exchanging some obvious signs we obtain similarly

$$
\limsup _{L \rightarrow \infty}\left(-\lambda_{j}^{-}\left(P_{L}\right)\right) \leq-\lambda_{j}^{-}\left(P_{\infty}\right) .
$$

\subsection{The infimum part of the proof of Theorem 4.1, We now prove}

$$
\liminf _{L \rightarrow \infty}\left( \pm \lambda_{j}^{ \pm}\left(P_{L}\right)\right) \geq \pm \lambda_{j}^{ \pm}\left(P_{\infty}\right) .
$$

We assume that we have a sequence $L_{i} \rightarrow \infty$, and that for each $i$ we have a system of orthogonal eigenvectors $\varphi_{i, 1}, \ldots, \varphi_{i, m}$ of $P_{L_{i}}$, i.e. $P_{L_{i}} \varphi_{i, \ell}=\lambda_{i, \ell} \varphi_{i, \ell}$ for $\ell \in\{1, \ldots, m\}$. Furthermore we suppose that $\lambda_{i, \ell} \rightarrow \bar{\lambda}_{\ell} \in\left(-\sigma_{P}, \sigma_{P}\right)$ for $\ell \in\{1, \ldots, m\}$.

Then

satisfies

$$
\psi_{i, \ell}:=\left(\frac{F_{L_{i}}}{F_{\infty}}\right)^{\frac{n-k}{2}} \varphi_{i, \ell}
$$

Furthermore

$$
P_{\infty} \psi_{i, \ell}=h_{i, \ell} \psi_{i, \ell} \quad \text { with } \quad h_{i, \ell}:=\left(\frac{F_{L_{i}}}{F_{\infty}}\right)^{k} \lambda_{i, \ell}
$$

$$
\left\|\psi_{i, \ell}\right\|_{L^{2}\left(M_{\infty}, g_{\infty}\right)}^{2}=\int_{M}\left(\frac{F_{L_{i}}}{F_{\infty}}\right)^{-k}\left|\varphi_{i, \ell}\right|^{2} d v^{g_{L_{i}}} \leq \sup _{M}\left|\varphi_{i, \ell}\right|^{2} \int_{M}\left(\frac{F_{L_{i}}}{F_{\infty}}\right)^{-k} d v^{g_{L_{i}}}
$$

Because of $\int_{M}\left(\frac{F_{L_{i}}}{F_{\infty}}\right)^{-k} d v^{g_{L}} \leq C \int r^{n-1-k} d r<\infty$ (for $n>k$ ) the norm $\left\|\psi_{i, \ell}\right\|_{L^{2}\left(M_{\infty}, g_{\infty}\right)}$ is finite as well, and we can renormalize such that

$$
\left\|\psi_{i, \ell}\right\|_{L^{2}\left(M_{\infty}, g_{\infty}\right)}=1 .
$$

Lemma 4.2. For any $\delta>0$ and any $\ell \in\{0, \ldots, m\}$ the sequence

is bounded.

$$
\left(\left\|\psi_{i, \ell}\right\|_{C^{k+1}\left(M \backslash B_{y}(\delta), g_{\infty}\right)}\right)_{i}
$$


Proof of the lemma. After removing finitely many $i$, we can assume that $\lambda_{i} \leq 2 \bar{\lambda}$ and $e^{-L_{i}}<\delta / 2$. Hence $F_{L}=F_{\infty}$ and $h_{i}=\lambda_{i}$ on $M \backslash B_{y}(\delta / 2)$. Because of

$$
\int_{M \backslash B_{y}(\delta / 2)}\left|\left(P_{\infty}\right)^{s} \psi_{i}\right|^{2} d v^{g_{\infty}} \leq(2 \bar{\lambda})^{2 s} \int_{M \backslash B_{y}(\delta / 2)}\left|\psi_{i}\right|^{2} d v^{g_{\infty}} \leq(2 \bar{\lambda})^{2 s}
$$

we obtain boundedness of $\psi_{i}$ in the Sobolev space $H^{s k}\left(M \backslash B_{y}(3 \delta / 4), g_{\infty}\right)$, and hence, for sufficiently large $s$ boudnedness in $C^{k+1}\left(M \backslash B_{y}(\delta), g_{\infty}\right)$. The lemma is proved.

Hence after passing to a subsequence $\psi_{i, \ell}$ converges in $C^{k, \alpha}\left(M \backslash B_{y}(\delta), g_{\infty}\right)$ to a solution $\bar{\psi}_{\ell}$ of

$$
P_{\infty} \bar{\psi}_{\ell}=\bar{\lambda}_{\ell} \bar{\psi}_{\ell} .
$$

By taking a diagonal sequence, one can obtain convergence in $C_{\mathrm{loc}}^{k, \alpha}\left(M_{\infty}\right)$ of $\psi_{i, \ell}$ to $\bar{\psi}_{\ell}$. It remains to prove that $\bar{\psi}_{1}, \ldots, \bar{\psi}_{m}$ are linearly independent, in particular that any $\bar{\psi}_{\ell} \neq 0$. For this we use the following lemma.

Lemma 4.3. For any $\varepsilon>0$ there is $\delta_{0}$ and $i_{0}$ such that

$$
\left\|\psi_{i, \ell}\right\|_{L^{2}\left(B_{y}\left(\delta_{0}\right), g_{\infty}\right)} \leq \varepsilon\left\|\psi_{i, \ell}\right\|_{L^{2}\left(M, g_{\infty}\right)}
$$

for all $i \geq i_{0}$ and all $\ell \in\{0, \ldots, m\}$. In particular,

$$
\left\|\psi_{i, \ell}\right\|_{L^{2}\left(M \backslash B_{y}\left(\delta_{0}\right), g_{\infty}\right)} \geq(1-\varepsilon)\left\|\psi_{i, \ell}\right\|_{L^{2}\left(M, g_{\infty}\right)} .
$$

Proof of the lemma. Because of Proposition 3.2 and

$$
\left\|P_{\infty} \psi_{i, \ell}\right\|_{L^{2}\left(M_{\infty}, g_{\infty}\right)} \leq\left|\bar{\lambda}_{\ell}\right|\left\|\psi_{i, \ell}\right\|_{L^{2}\left(M_{\infty}, g_{\infty}\right)}=\left|\bar{\lambda}_{\ell}\right|
$$

we get

$$
\left\|\left(\nabla^{\infty}\right)^{s} \psi_{i, \ell}\right\|_{L^{2}\left(M_{\infty}, g_{\infty}\right)} \leq C
$$

for all $s \in\{0, \ldots, k\}$. Let $\chi$ be a cut-off dunction as in Subsection 4.2 with $T=-\log \delta$. Hence

$$
\left\|P_{\infty}\left((1-\chi) \psi_{i, \ell}\right)-(1-\chi) P_{\infty}\left(\psi_{i, \ell}\right)\right\|_{L^{2}\left(M_{\infty}, g_{\infty}\right)} \leq \frac{C}{T}=\frac{C}{-\log \delta} .
$$

On the other hand $\left(B_{y}(\delta) \backslash\{y\}, g_{\infty}\right)$ converges for $\delta \rightarrow 0$ to $\mathbb{S}^{n-1} \times(0, \infty)$ in the $C^{\infty}$-topology. Hence there is a function $\tau(\delta)$ converging to 0 such that

$$
\left\|P_{\infty}\left((1-\chi) \psi_{i, \ell}\right)\right\|_{L^{2}\left(M_{\infty}, g_{\infty}\right)} \geq\left(\sigma_{p}-\tau(\delta)\right)\left\|(1-\chi) \psi_{i, \ell}\right\|_{L^{2}\left(M_{\infty}, g_{\infty}\right)} .
$$

Using the obvious relation

$$
\left\|(1-\chi) P_{\infty}\left(\psi_{i, \ell}\right)\right\|_{L^{2}\left(M_{\infty}, g_{\infty}\right)} \leq\left|\lambda_{i, \ell}\right|\left\|(1-\chi) \psi_{i, \ell}\right\|_{L^{2}\left(M_{\infty}, g_{\infty}\right)}
$$

we obtain with (13) and (14)

$$
\left\|\psi_{i, \ell}\right\|_{L^{2}\left(B_{y}\left(\delta^{2}\right), g_{\infty}\right)} \leq\left\|(1-\chi) \psi_{i, \ell}\right\|_{L^{2}\left(M_{\infty}, g_{\infty}\right)} \leq \frac{C}{|\log \delta|\left(\sigma_{P}-\tau(\delta)-\left|\lambda_{i, \ell}\right|\right)} .
$$

The right hand side is smaller than $\varepsilon$ for $i$ sufficiently large and $\delta$ sufficiently small. The main statement of the lemma then follows for $\delta_{0}:=\delta^{2}$. The Minkowski inequality yields.

$$
\left\|\psi_{i, \ell}\right\|_{L^{2}\left(M \backslash B_{y}\left(\delta^{2}\right), g_{\infty}\right)} \geq 1-\left\|\psi_{i, \ell}\right\|_{L^{2}\left(B_{y}\left(\delta^{2}\right), g_{\infty}\right)} \geq 1-\varepsilon .
$$

The convergence in $C^{1}\left(M \backslash B_{y}\left(\delta_{0}\right)\right)$ implies strong convergence in $L^{2}\left(M \backslash B_{y}\left(\delta_{0}\right), g_{\infty}\right)$ of $\psi_{i, \ell}$ to $\bar{\psi}_{\ell}$. Hence

$$
\left\|\bar{\psi}_{\ell}\right\|_{L^{2}\left(M \backslash B_{y}\left(\delta_{0}\right), g_{\infty}\right)} \geq 1-\varepsilon
$$

and thus $\left\|\bar{\psi}_{\ell}\right\|_{L^{2}\left(M_{\infty}, g_{\infty}\right)}=1$. The orthogonality of these sections is provided by the following lemma, and the inequality (12) then follows immediatly.

Lemma 4.4. The sections $\bar{\psi}_{1}, \ldots, \bar{\psi}_{m}$ are orthogonal. 
Proof of the lemma. The sections $\varphi_{i, 1}, \ldots, \varphi_{i, \ell}$ are orthogonal. For any fixed $\delta_{0}$ (given by the previous lemma), it follows for sufficiently large $i$ that

$$
\begin{aligned}
\left|\int_{M \backslash B_{y}\left(\delta_{0}\right)}\left\langle\psi_{i, \ell}, \psi_{i, \tilde{\ell}}\right\rangle d v^{g_{\infty}}\right| & =\left|\int_{M \backslash B_{y}\left(\delta_{0}\right)}\left\langle\varphi_{i, \ell}, \varphi_{i, \tilde{\ell}}\right\rangle d v^{g_{L_{i}}}\right| \\
& =\left|\int_{B_{y}\left(\delta_{0}\right)}\left\langle\varphi_{i, \ell}, \varphi_{i, \tilde{\ell}}\right\rangle d v^{g_{L_{i}}}\right| \\
& =|\int_{B_{y}\left(\delta_{0}\right)} \underbrace{\left(\frac{F_{L_{i}}}{F_{\infty}}\right)^{k}}_{\leq 1}\left\langle\psi_{i, \ell}, \psi_{i, \tilde{\ell}}\right\rangle d v^{g_{\infty}}| \\
& \leq \varepsilon^{2}
\end{aligned}
$$

Because of strong $L^{2}$ convergence on $M \backslash B_{y}\left(\delta_{0}\right)$ this implies

$$
\left|\int_{M \backslash B_{y}\left(\delta_{0}\right)}\left\langle\bar{\psi}_{\ell}, \bar{\psi}_{\hat{\ell}}\right\rangle d v^{g_{\infty}}\right| \leq \varepsilon^{2}
$$

for $\tilde{\ell} \neq \ell$, and hence in the limit $\varepsilon \rightarrow 0$ (and $\delta_{0} \rightarrow 0$ ) we get the orthogonality of $\bar{\psi}_{1}, \ldots, \bar{\psi}_{m}$.

\section{REFERENCES}

[1] B. Ammann, Spin-Strukturen und das Spektrum des Dirac-Operators, Ph.D. thesis, University of Freiburg, Germany, 1998, Shaker-Verlag Aachen 1998, ISBN 3-8265-4282-7.

[2] — The smallest Dirac eigenvalue in a spin-conformal class and cmc-immersions, Preprint, 2003.

[3] _ A spin-conformal lower bound of the first positive Dirac eigenvalue, Diff. Geom. Appl. 18 (2003), 21-32.

[4] — A variational problem in conformal spin geometry, Habilitationsschrift, Universität Hamburg, 2003.

[5] B. Ammann and C. Bär, Dirac eigenvalues and total scalar curvature, J. Geom. Phys. 33 (2000), 229-234.

[6] B. Ammann and E. Humbert, The first conformal Dirac eigenvalue on 2-dimensional tori, J. Geom. Phys. 56 (2006), 623-642.

[7] B. Ammann, E. Humbert, and B. Morel, Mass endomorphism and spinorial Yamabe type problems, Comm. Anal. Geom. 14 (2006), 163-182.

[8] B. Ammann, A. D. Ionescu, and V. Nistor, Sobolev spaces on Lie manifolds and regularity for polyhedral domains, Doc. Math. 11 (2006), 161-206.

[9] B. Ammann, R. Lauter, and V. Nistor, On the geometry of Riemannian manifolds with a Lie structure at infinity, Int. J. Math. Math. Sci. (2004), no. 1-4, 161-193.

[10] _ Pseudodifferential operators on manifolds with a Lie structure at infinity, Ann. of Math. 165 (2007), 717-747.

[11] C. Bär, The Dirac operator on space forms of positive curvature, J. Math. Soc. Japan 48 (1996), 69-83.

[12] H. Baum, Spin-Strukturen und Dirac-Operatoren über pseudoriemannschen Mannigfaltigkeiten, Teubner Verlag, 1981.

[13] A. L. Besse, Einstein manifolds, Ergebnisse der Mathematik und ihrer Grenzgebiete, 3. Folge, no. 10, Springer-Verlag, 1987.

[14] T. P. Branson, Differential operators canonically associated to a conformal structure, Math. Scand. 57 (1985), no. 2, 293-345.

[15] _ Group representations arising from Lorentz conformal geometry, J. Funct. Anal. 74 (1987), no. 2, $199-291$.

[16] - Second order conformal covariants, Proc. Amer. Math. Soc. 126 (1998), no. 4, 1031-1042.

[17] B. Colbois and A. El Soufi, Eigenvalues of the Laplacian acting on p-forms and metric conformal deformations, Proc. of Am. Math. Soc. 134 (2006), no. 3, 715-721.

[18] A. El Soufi and S. Ilias, Immersions minimales, première valeur propre du laplacien et volume conforme, Math. Ann. 275 (1986), no. 2, 257-267.

[19] H. D. Fegan, Conformally invariant first order differential operators., Quart. J. Math. Oxford, II. series 27 (1976), $371-378$.

[20] R. Gover and L. J. Peterson, Conformally invariant powers of the Laplacian, Q-curvature, and tractor calculus, Comm. Math. Phys. 235 (2003), no. 2, 339-378.

[21] E. Hebey and F. Robert, Coercivity and Struwe's compactness for Paneitz type operators with constant coefficients, Calc. Var. Partial Differential Equations 13 (2001), no. 4, 491-517.

[22] O. Hijazi, Spectral properties of the Dirac operator and geometrical structures., Ocampo, Hernan (ed.) et al., Geometric methods for quantum field theory. Proceedings of the summer school, Villa de Leyva, Colombia, July 12-30, 1999. Singapore: World Scientific. 116-169, 2001.

[23] N. Hitchin, Harmonic spinors, Adv. Math. 14 (1974), 1-55.

[24] P. Jammes, Extrema de valeurs propres dans une classe conforme, Sémin. Théor. Spectr. Géom. 24 (2007), $23-42$. 
[25] I. Kolář, P. W. Michor, and J. Slovák, Natural operations in differential geometry, Springer-Verlag, Berlin, 1993.

[26] N. Korevaar, Upper bounds for eigenvalues of conformal metrics, J. Differ. Geom. 37 (1993), no. 1, 73-93.

[27] S. Mac Lane, Categories for the working mathematician, Graduate Texts in Mathematics, vol. 5, Springer-Verlag, New York, 1998.

[28] R. B. Melrose, The Atiyah-Patodi-Singer index theorem, Research Notes in Mathematics, vol. 4, A K Peters Ltd., Wellesley, MA, 1993.

Authors' address:

Bernd Ammann

Institut Élie Cartan BP 239

Université de Nancy 1

54506 Vandoeuvre-lès -Nancy Cedex

France

Pierre Jammes

Laboratoire d'analyse non linéaire et géométrie

Université d'Avignon

33 rue Louis Pasteur

84000 Avignon

France

E-Mail:

bernd.ammann at gmx.net and pierre.jammes at univ-avignon.fr 